\title{
Water waves over a strongly undulating bottom
}

\author{
V. P. Ruban* \\ Landau Institute for Theoretical Physics, 2 Kosygin Street, 119334 Moscow, Russia
}

(Dated: July 19, 2018)

\begin{abstract}
Two-dimensional free-surface potential flows of an ideal fluid over a strongly inhomogeneous bottom are investigated with the help of conformal mappings. Weakly-nonlinear and exact nonlinear equations of motion are derived by the variational method for arbitrary seabed shape parameterized by an analytical function. As applications of this theory, band structure of linear waves over periodic bottoms is calculated and evolution of a strong solitary wave running from a deep region to a shallow region is numerically simulated.

PACS numbers: $47.15 . \mathrm{Hg}, 47.35 .+\mathrm{i}, 47.10 .+\mathrm{g}$
\end{abstract}

\section{INTRODUCTION}

The classical problem of water waves over a variable seabed has attracted much attention (see 1, 2, 3, 4, 5, $6,7,8,9,10,11,12,13,14,15,16,17,18,19,20,121$, $22,23,24,25,26,27,[28,[29,30]$ and references therein). There are some significant differences in this interesting and practically important problem, as compared to the theory of waves on a deep water or in canals with a flat horizontal bottom. In situations where the fluid depth is less or of the same order as a typical length of surface wave, inhomogeneity of the bottom is a reason for linear and nonlinear wave scattering and transformation, and it strongly affects wave propagation. These phenomena occur so widely that one can meet them almost everywhere, although with different scales. Examples of strongly nonlinear dynamics are ocean waves running on a beach, or motion of disturbed water in a puddle after a car. Among linear effects due to bottom topography is existence of special edge-localized waves discovered by Stokes [17, 18, 19, 20], that propagate along the shore line of a beach. Over an axially symmetric underwater hill, quasi-localized wave modes with non-zero angular momentum can exist, similar to long-life-time states of a quantum particle confined by a potential barrier of a finite width 6, 31]. It is necessary to say that underwater obstacles of definite shapes and sizes can serve as waveguides (a narrow and long underwater crest) or as lenses (an oblong underwater hill oriented crosswise to the wave propagation). A qualitative explanation for all the linear effects is simple. Indeed, let $\mathbf{r}_{\perp}$ be the coordinate in the horizontal plane, $H\left(\mathbf{r}_{\perp}\right)$ the depth corresponding to quiet surface. Then, looking at the well known dispersion relation for small-amplitude gravitational surface waves,

$$
\omega(K, H)=\sqrt{g K \tanh (K H)}
$$

(where $\omega$ is the frequency, $K$ is the absolute value of the wave vector, $g$ is the gravitational acceleration), one can see that the local refraction index $n\left(\omega, \mathbf{r}_{\perp}\right)$ increases

*Electronic address: ruban@itp.ac.ru as the depth $H\left(\mathbf{r}_{\perp}\right)$ decreases, in accordance with the formulas

$$
\begin{aligned}
n\left(\omega, H\left(\mathbf{r}_{\perp}\right)\right) \equiv \frac{K\left(\omega, H\left(\mathbf{r}_{\perp}\right)\right)}{K(\omega, H=\infty)} & =\frac{g K\left(\omega, H\left(\mathbf{r}_{\perp}\right)\right)}{\omega^{2}}>1 \\
\frac{\partial K(\omega, H)}{\partial H} & <0
\end{aligned}
$$

where the function $K(\omega, H)$ is determined by Eq. (1). Therefore, as in the conventional light optics, here an oblique wave changes its direction of propagation when meets gradient of $n$. Also, the total internal reflection is possible in propagation from smaller depth to larger depth.

Besides observing such natural phenomena, a set of laboratory experiments has been carried out to investigate various aspects of the given problem in more idealized and controlled conditions than are achieved in nature 9, 12, 13, 14, 19, 20, 27]. In particular, waves over locally periodic bottoms were studied experimentally [9, 12, 13, 14, 27], and such a general for periodic media effect was observed as the Bragg resonances and the corresponding band structure with gaps in wave spectrum. It is worth to say that in natural conditions quasiperiodic sand bars occur quite often.

In general, a qualitative picture of the mentioned phenomena is clear. As concerning the quantitative side of the mathematical theory of waves over a variable bottom, here not everything that necessary has been done, because practically all developed up to now analytical models and methods are related to the limit cases where the fluid is considered as ideal, and the slope of the bottom is small (or amplitude of the bottom undulations is small). For the general three-dimensional (3D) Hamiltonian theory of water waves, such restriction seems to be unavoidable even in considering the most simple, irrotational flows when the state of the system is described by a minimal set of functions, namely by a pair of canonically conjugated quantities as the deviation $\eta\left(\mathbf{r}_{\perp}, t\right)$ of the free surface from the horizontal plane and the boundary value $\psi\left(\mathbf{r}_{\perp}, t\right)$ of the velocity potential 32,33$]$. A technical difficulty exists here that, when working in $3 \mathrm{D}$ space, it is impossible to represent in convenient and compact form 
the kinetic energy functional $\mathcal{K}\{\eta, \psi\}$ which is part of the Hamiltonian of the system. Small values of the bottom slope and of the free surface slope make possible expansion of the Hamiltonian to asymptotic series and subsequent application of various variants of the perturbation theory. In such traditional approach, an inhomogeneous bottom does not allow to write in exact form even linearized equations, not speaking about nonlinear corrections.

There are more favorable conditions for progress in theory of 2D potential ideal flows with a free boundary, and the reason for this is the possibility to employ such powerful mathematical tools as analytical functions and the corresponding conformal mappings. Time-dependent conformal mappings were successfully used for studying strongly nonlinear 2D wave dynamics on deep water and over straight horizontal bottom 34, 35, 36, 37, 38, 39, 40, 41]. In the cited works the region occupied by resting fluid (the lower half-plane or a horizontal stripe) was mapped onto the region with disturbed free boundary, and the real axis was transformed into moving boundary. Such a conformal "straightening" of free surface has provided a compact representation for the Hamiltonian, derivation of exact equations of motion, and possibility for precise numerical simulations of the system evolution.

The purpose of this work is to study the effect of a strongly undulating bottom on 2D ideal potential flows with a free surface. Here conformal mappings are used as well, and this is done in two variants. In the first, "moderate" variant (Sec. 2), a fixed conformal mapping "straightens" the bottom, but not the free boundary. More exactly: instead of the Cartesian coordinates $x$ and $y$ (with $y$-axis up-directed), curvilinear coordinates $u$ and $v$ are introduced, and the change of coordinates is performed with the help of an analytical function $z(w)$ which maps the stripe $-1<\operatorname{Im} w<0$ onto the region between the horizontal line $y=0$ and the inhomogeneous bottom $y=-H(x)$. In this case $x+i y=z(u+i v)$, the horizontal line $y=0$ corresponds to $v=0$, and on the bottom $v=-1$. The bottom may have arbitrary large slope and even impending pieces where the dependence $H(x)$ is multi-valued, as shown in Fig 1 The shape of free surface will be described by a function $v=V(u, t)$. The Lagrangian for weakly-nonlinear waves is represented as an integral series in powers of the dynamical variables $V(u, t)$ and $\psi(u, t)$, with coefficients explicitly depending on the spatial coordinate $u$. In the small-amplitude limit, the wave dynamics is governed by linear integraldifferential equations. It is using the conformal variables $u$ and $v$, that allows us to obtain these equations in exact form, contrary to the traditional approach where even linearized equations can be obtained only approximately by expansion in the small parameter, the slope of the bottom. The definition "moderate" for this variant emphasizes that straightening of the bottom without straightening the free boundary is able to provide not more than a weakly-nonlinear theory. Nevertheless, such
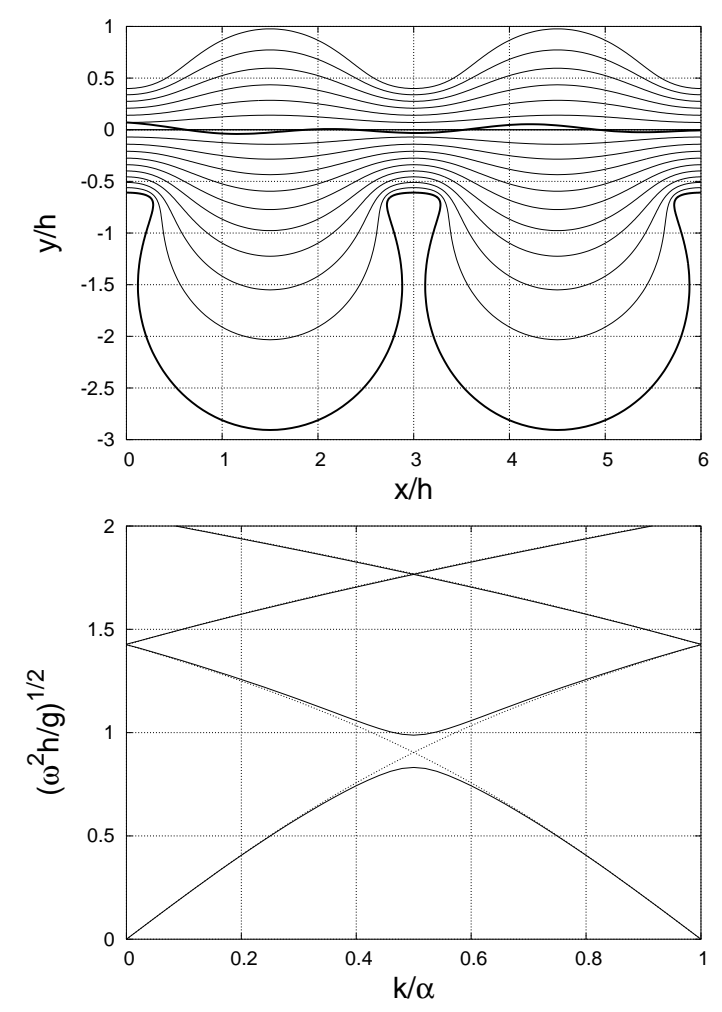

FIG. 1: Left: periodic shape of the bottom (lower thick line), levels of constant $v=-0.9,-0.8, \ldots,+0.6$ (thin lines), and (schematically) free boundary (thick line near $y=0$ ). Right: the corresponding band structure of the spectrum of linear waves. In this example $z(w) / h=w+(2 \epsilon / \alpha) \sin (\alpha w) /(1+$ $b \cos (\alpha w))$, with the parameters $\alpha=2 \pi / 3, \epsilon=-0.17, b=$ 0.16 .

a theory seems to be helpful and applicable in many practical cases when wave amplitude is small. The results of this part of the work are the derivation of the Hamiltonian functional for weakly-nonlinear potential surface waves in canals having arbitrary bottom shape, as well as calculations for band structure of spectrum for a number of periodic bottom profiles. As an example how to treat the linearized equations, also the problem is considered of wave reflection on a smooth "step" - changing the depth from $h_{1}$ to $h_{2}$.

The other variant of using the conformal mappings may be called "radical" in the sense it is valid for arbitrary shape of the bottom and for arbitrary shape of the free surface. It is an exact combined theory where a time-dependent conformal mapping straightens both the bottom and the free boundary (Sec. 3). Such a mapping can be represented as the result of two mappings: $x+i y=$ $z(\zeta(w, t))$, where the first function $\zeta(w, t)$ maps the horizontal stripe $-1<v<0$ onto the region $D_{\zeta}(t)$ with the straight lower boundary $(\operatorname{Im} \zeta=-1)$ and with a perturbed upper boundary, after that the time-independent function $z(\zeta)$ maps the half-plane $\operatorname{Im} \zeta>-1$ onto the region $y>-H(x)$ in the physical plane bounded from be- 
low by the bottom. The shape of the free surface will be described by the formula $X+i Y=Z(u, t)=z(\zeta(u, t))$. However, it appears that exact nonlinear equations for $Z(u, t)$ in the inhomogeneous case have the same form as the known equations for waves over a horizontal bottom [38], but with different analyticity requirements imposed on the solutions. Numerical solutions obtained by the spectral method are presented that describe a running and breaking wave (Sec. 4).

\section{WEAKLY-NONLINEAR THEORY}

So, suppose we know the analytical function $z(w)=$ $x(u, v)+i y(u, v)$ which maps the horizontal stripe $-1<$ $\operatorname{Im} w<0$ onto the region occupied by the fluid at rest, and this function takes real values on the real axis: $z(u)=x(u, 0)$. The velocity field is irrotational, and the velocity potential $\varphi(u, v)$ satisfies the Laplace equation $\varphi_{u u}+\varphi_{v v}=0$ in the flow region $-1<v<V(u, t)$, with the boundary conditions $\left.\varphi_{v}\right|_{v=-1}=0,\left.\varphi\right|_{v=V(u)}=\psi(u)$. Due to conformal invariance of the Laplace equation in $2 \mathrm{D}$-space, hence equation $\varphi_{x x}+\varphi_{y y}=0$ is satisfied as well, with no-penetration boundary condition on the bottom: $\partial \varphi /\left.\partial n\right|_{y=-H(x)}=0$. Let us now take into account the fact that the Lagrangian functional for potential surface waves has the following structure [32, 33, 38]:

$$
\mathcal{L}=\int \psi \dot{\eta} d x-\mathcal{H}=\int \psi\left(Y_{t} X_{u}-Y_{u} X_{t}\right) d u-\mathcal{H},
$$

where $Y(u, t)=y(u, V(u, t)), X(u, t)=x(u, V(u, t))$, and the Hamiltonian functional $\mathcal{H}$ is the total energy of the system - sum of the kinetic energy and the potential energy in gravitational field (in this paper we neglect surface tension effects, though they can be easily incorporated by adding to the Hamiltonian the surface energy). In our variables

$$
\begin{aligned}
\mathcal{H} & =\frac{1}{2} \int d u \int_{-1}^{V(u)}\left(\varphi_{u}^{2}+\varphi_{v}^{2}\right) d v \\
& +\frac{g}{2} \int y^{2}(u, V(u)) \frac{d}{d u} x(u, V(u)) d u .
\end{aligned}
$$

This system has the obvious stable equilibrium $\psi=0$, $V=0$, hence one may consider weak oscillations near this equilibrium state. In a standard way (see, for instance [33]), let us expand the Lagrangian (3) in powers of the dynamical variables $\psi$ and $V$. It is clear that due to the symmetry principle the expansion for $y(u, v)$ contains only the odd powers of $v$, while the expansion for $x(u, v)$ contains only the even powers of $v$. Therefore up to the third order in powers of $\psi$ and $V$ the Lagrangian (3) is equal to

$\tilde{\mathcal{L}}=\int \psi V_{t} x^{\prime 2}(u) d u-\mathcal{K}^{(2)}\{\psi\}-\mathcal{K}^{(3)}\{\psi, V\}-\mathcal{P}^{(2)}\{V\}$, where $x^{\prime}(u)=z^{\prime}(u+0 i)=x_{u}(u, 0)$, and the equality $y_{v}=$ $x_{u}$ has been taken into account in the first integral in r.h.s. The expansion for the kinetic energy (calculation of the functionals $\mathcal{K}^{(2)}$ and $\left.\mathcal{K}^{(3)}\right)$ is performed in a standard manner [33, 38] and gives

$$
\begin{gathered}
\mathcal{K}^{(2)}\{\psi\}=\frac{1}{2} \int \psi[\hat{k} \tanh \hat{k}] \psi d u, \\
\mathcal{K}^{(3)}\{\psi, V\}=\frac{1}{2} \int\left[\psi_{u}^{2}-([\hat{k} \tanh \hat{k}] \psi)^{2}\right] V d u .
\end{gathered}
$$

Here the linear Hermitian operator $[\hat{k} \tanh \hat{k}]$ has been introduced, acting as

$$
[\hat{k} \tanh \hat{k}] \psi(u)=-\mathrm{P} . \mathrm{V} \cdot \int_{-\infty}^{+\infty} \frac{\psi_{\tilde{u}}(\tilde{u}) d \tilde{u}}{2 \sinh [(\pi / 2)(\tilde{u}-u)]} .
$$

In Fourier-representation this operator simply multiplies the Fourier-harmonics $\psi_{k}=\int \psi(u) \exp (-i k u) d u$ by $k \tanh k$. Quadratic on $V$ part of the potential energy is

$$
\mathcal{P}^{(2)}\{V\}=\frac{g}{2} \int V^{2} x^{\prime 3}(u) d u .
$$

It is convenient to deal with the function $\xi(u, t)=$ $V(u, t) x^{\prime 2}(u)$ canonically conjugated to $\psi(u, t)$, and write the corresponding up-to-third-order Hamiltonian in terms of $\xi$ and $\psi$ :

$$
\begin{aligned}
\tilde{\mathcal{H}}\{\xi, \psi\} & =\frac{1}{2} \int \psi[\hat{k} \tanh \hat{k}] \psi d u+\frac{g}{2} \int \frac{\xi^{2}}{x^{\prime}(u)} d u \\
& +\frac{1}{2} \int \frac{\left[\psi_{u}^{2}-([\hat{k} \tanh \hat{k}] \psi)^{2}\right] \xi}{x^{\prime 2}(u)} d u .
\end{aligned}
$$

Physically, this asymptotic expansion of the Hamiltonian is on a small parameter - the slope of the free surface (see 33. for more comments and references). The weaklynonlinear equations of motion have the standard Hamiltonian structure

$$
\begin{gathered}
\xi_{t}=\frac{\delta \tilde{\mathcal{H}}}{\delta \psi}=[\hat{k} \tanh \hat{k}] \psi-\frac{\partial}{\partial u}\left(\frac{\xi \psi_{u}}{x^{\prime 2}(u)}\right) \\
-[\hat{k} \tanh \hat{k}]\left(\frac{\xi[\hat{k} \tanh \hat{k}] \psi}{x^{\prime 2}(u)}\right), \\
-\psi_{t}=\frac{\delta \tilde{\mathcal{H}}}{\delta \xi}=g \frac{\xi}{x^{\prime}(u)}+\frac{\left[\psi_{u}^{2}-([\hat{k} \tanh \hat{k}] \psi)^{2}\right]}{2 x^{\prime 2}(u)} .
\end{gathered}
$$

If $\left|x^{\prime \prime}(u) / x^{\prime}(u)\right| \ll 1$, then $x^{\prime}(u)$ is approximately equal to the equilibrium depth $H(u)$. For long waves over a such slowly varying bottom, only Fourier-harmonics $\psi_{k}$ and $\xi_{k}$ with small $k$ are excited, so in this case the Hamiltonian (10) can be simplified to the local form

$\tilde{\mathcal{H}}_{1}=\int\left[\frac{\psi_{u}^{2}}{2}-\frac{\psi_{u u}^{2}}{6}+\frac{2 \psi_{u u u}^{2}}{15}+\frac{g \xi^{2}}{2 x^{\prime}(u)}+\frac{\xi\left[\psi_{u}^{2}-\psi_{u u}^{2}\right]}{2 x^{\prime 2}(u)}\right] d u$,

which is suitable for consideration of such phenomena as interaction of solitons with the bottom topography. 


\section{A. Linearized equations}

Now let us consider the linearized system

$$
\xi_{t}=[\hat{k} \tanh \hat{k}] \psi, \quad-\psi_{t}=g \frac{\xi}{x^{\prime}(u)} .
$$

For a monochromatic wave $(\xi, \psi \propto \exp (-i \omega t))$ Eqs. (14) are reduced to the single integral equation

$$
\left(\frac{\omega^{2}}{g} x^{\prime}(u)-\hat{k} \tanh \hat{k}\right) \psi_{\omega}(u)=0 .
$$

In the low-frequency limit this equation can be considerably simplified. A variant of simplification is to introduce a new function $f$ by the equality $\hat{k} \tanh \hat{k} \psi_{\omega}(u)=-f_{u u}$. Then we obtain the equation

$$
\left(\frac{\omega^{2}}{g} x^{\prime}(u) \hat{k} \operatorname{coth} \hat{k}+(d / d u)^{2}\right) f=0 .
$$

The low-frequency limit corresponds to long wavelengths, when $\hat{k} \operatorname{coth} \hat{k} \approx 1+\hat{k}^{2} / 3=1-(1 / 3)(d / d u)^{2}$, so we have to deal with the second-order differential equation

$$
f_{u u}(u)+\frac{\frac{\omega^{2}}{g} x^{\prime}(u)}{1-\frac{\omega^{2} x^{\prime}(u)}{3 g}} f(u)=0 .
$$

where $\omega^{2} x^{\prime}(u) / g$ should be small (only in this case the wave length is indeed effectively long; remember that $x^{\prime}(u)$ is of the same order as the depth).

Higher-order approximations to equation (15) can be derived in a similar manner, for instance by change $\psi_{\omega}(u)=[\cosh \hat{k}] f(u)$ and subsequent expanding $[\cosh \hat{k}]$ and $[\hat{k} \sinh \hat{k}]$ in powers of $\hat{k}^{2}=-(d / d u)^{2}$.

As an explicit example of using Eq.(17), we consider reflection of a long wave from a step-shaped bottom inhomogeneity described by the function

$$
z(w)=h_{1} w+\frac{\left(h_{2}-h_{1}\right)}{\alpha} \ln \left(1+e^{\alpha w}\right),
$$

where $h_{1}>h_{2}>0,0<\alpha \ll \pi$. If frequency of the wave is small, $\omega \ll \sqrt{g / h_{1}}$, then equation (17) may be applied. Calculating the derivative

$$
z^{\prime}(w)=h_{1}+\left(h_{2}-h_{1}\right) \frac{1}{1+e^{-\alpha w}}=\frac{h_{1} e^{-\alpha w}+h_{2}}{e^{-\alpha w}+1},
$$

we have for $f(u)$ the equation

$$
f_{u u}(u)+\frac{\omega^{2}}{g} \frac{\left[\tilde{h}_{1} C e^{-\alpha u}+\tilde{h}_{2}\right]}{\left[C e^{-\alpha u}+1\right]} f(u)=0,
$$

where

$$
\tilde{h}_{1}=\frac{h_{1}}{1-\frac{\omega^{2} h_{1}}{3 g}}, \quad \tilde{h}_{2}=\frac{h_{2}}{1-\frac{\omega^{2} h_{2}}{3 g}}, \quad C=\frac{1-\frac{\omega^{2} h_{1}}{3 g}}{1-\frac{\omega^{2} h_{2}}{3 g}} .
$$

A general solution for equation (20) is known [31]. In particular, the reflection coefficient is given by the expression

$$
R(\omega)=\left(\frac{\sinh \left[\frac{\pi \omega}{\alpha \sqrt{g}}\left(\sqrt{\tilde{h}_{1}}-\sqrt{\tilde{h}_{2}}\right)\right]}{\sinh \left[\frac{\pi \omega}{\alpha \sqrt{g}}\left(\sqrt{\tilde{h}_{1}}+\sqrt{\tilde{h}_{2}}\right)\right]}\right)^{2} .
$$

\section{B. Periodic bottom: The band structure of the spectrum}

Interesting phenomena occur if shape of the bottom is periodic:

$$
z^{\prime}(w)=h \sum_{n} a_{n} \exp (\text { in } \alpha w), \quad a_{-n}=\bar{a}_{n} .
$$

Here $h$ is a dimensional parameter, $a_{n}$ are some complex Fourier-coefficients. Obviously, $x^{\prime}(u)=z^{\prime}(u)>0$ and $\left|a_{n}\right|$ decay rapidly at large $|n|$, since $z^{\prime}(w)$ does not have any singularities at $-1<\operatorname{Im} w<1$. The equation (15) for eigen-functions $\psi_{\lambda}(u)$ (where $\lambda=\omega^{2} h / g$ ) now has the form

$$
\lambda\left(\sum_{n} a_{n} \exp (i n \alpha u)\right) \psi(u)-[\hat{k} \tanh \hat{k}] \psi(u)=0,
$$

or in Fourier-representation

$$
\lambda \sum_{n} a_{n} \psi_{k-n \alpha}=k \tanh k \psi_{k}
$$

For convenience let us denote

$$
F_{\nu}=\alpha \nu \tanh (\alpha \nu), \quad \Psi_{\nu}=\psi_{\alpha \nu}
$$

Now we have the infinite chain of linear equations

$$
\lambda \sum_{n} a_{n} \Psi_{\nu-n}=F_{\nu} \Psi_{\nu}
$$

where $\Psi_{\nu_{1}}$ and $\Psi_{\nu_{2}}$ interact if the difference between $\nu_{1}$ and $\nu_{2}$ is an integer number. Let us fix some $\nu$. Nontrivial solutions of the system (27) exist only at definite values $\lambda=\lambda_{m}(\nu)$, where $m=1,2,3, \ldots$ It is necessary to note that the functions $\lambda_{m}(\nu)$ are periodic: $\lambda_{m}(\nu+1)=\lambda_{m}(\nu)$, and even: $\lambda_{m}(-\nu)=\lambda_{m}(\nu)$. This determines the band structure of the spectrum with frequency gaps (see Figs 12). For numerical computing $\lambda_{m}(\nu)$ it is necessary to cut the infinite chain (27) at some large but finite length, thus considering only $\nu$ between $-N$ and $N$. Practically $N$ should be several times larger than the index $m$ of $\lambda_{m}$. Numerical results for $\sqrt{\lambda}_{m}$ shown in the figures 12 were obtained with the help of the mathematical package Maple 8 taking $N=10$.

Fig 2 shows that in some cases even for strongly undulating bottom the coefficients $a_{n}$ with $n \geq 1$ can be still small $\left(a_{1}=\epsilon=0.2 \ll 1\right)$. In these cases it is easy to calculate analytically in the main approximation positions 

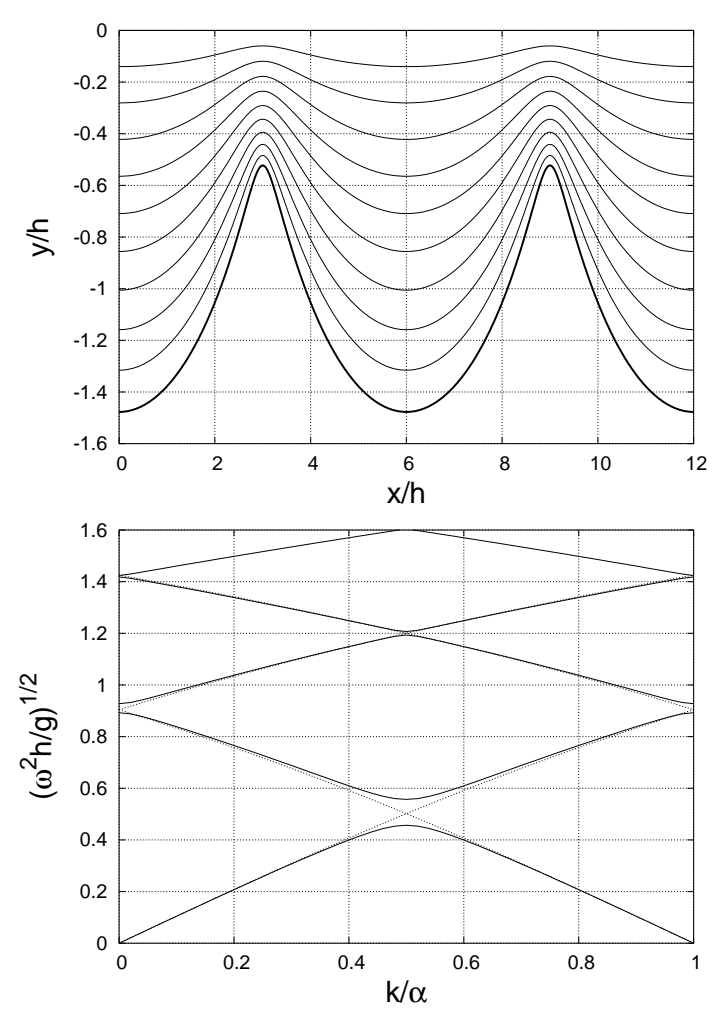

FIG. 2: Shape of the bottom, levels of constant $v$, and the band structure for $z(w) / h=w+(2 \epsilon / \alpha) \sin (\alpha w)$, with $\alpha=$ $\pi / 3, \epsilon=0.2$.

of the gaps. For example, let us consider the bottom profile as in Fig 2] where $x^{\prime}(u)=h(1+2 \epsilon \cos (\alpha u))$. The gaps in spectrum correspond to integer or half-integer $\nu$ 's. It is important that at these values of $\nu$, solutions of the linear chain (27) possess definite parity, in the sense that $\Psi_{-\nu}= \pm \Psi_{\nu}$. This allows us in gap calculation consider only positive $\nu$. Let us first consider half-integer $\nu$ 's and the corresponding half-infinite chain

$$
\begin{gathered}
\left(\lambda-F_{1 / 2}\right) \Psi_{1 / 2}+\lambda \epsilon\left( \pm \Psi_{1 / 2}+\Psi_{3 / 2}\right)=0 \\
\left(\lambda-F_{3 / 2}\right) \Psi_{3 / 2}+\lambda \epsilon\left(\Psi_{1 / 2}+\Psi_{5 / 2}\right)=0 \\
\left(\lambda-F_{5 / 2}\right) \Psi_{5 / 2}+\lambda \epsilon\left(\Psi_{3 / 2}+\Psi_{7 / 2}\right)=0 \\
\cdots
\end{gathered}
$$

Obviously, the even and odd cases result in different $\lambda$ 's, and it is this difference that determines the gaps in spectrum. For main-order calculation of the first and third gaps, we cut this chain: $\Psi_{7 / 2}=0, \Psi_{9 / 2}=0$, and so on. Now we have to solve the equation for zeros of the determinant $3 \times 3$

$$
\begin{array}{r}
{\left[\left\{\lambda(1 \pm \epsilon)-F_{1 / 2}\right\}\left(\lambda-F_{3 / 2}\right)-\lambda^{2} \epsilon^{2}\right]\left(\lambda-F_{5 / 2}\right)} \\
-\lambda^{2} \epsilon^{2}\left[\lambda(1 \pm \epsilon)-F_{1 / 2}\right]=0
\end{array}
$$

First we take $\lambda=F_{1 / 2}+\Delta_{1}$, where $\Delta_{1}$ is a small quantity of the order $\epsilon$. In the main order $\Delta_{1} \pm \epsilon F_{1 / 2}=0$, and this gives us the first gap: $F_{1 / 2}(1-\epsilon)<\lambda<F_{1 / 2}(1+\epsilon)$.
For the third gap we write $\lambda=F_{3 / 2}+\Delta_{3}$, where $\Delta_{3}$ is of order $\epsilon^{2}$. The equation for $\Delta_{3}$ with the third order accuracy is

$$
\begin{array}{r}
{\left[\left(F_{3 / 2}(1 \pm \epsilon)-F_{1 / 2}\right) \Delta_{3}-\epsilon^{2} F_{3 / 2}^{2}\right]\left(F_{3 / 2}-F_{5 / 2}\right)} \\
-\epsilon^{2} F_{3 / 2}^{2}\left(F_{3 / 2}(1 \pm \epsilon)-F_{1 / 2}\right)=0
\end{array}
$$

From here we find

$$
\Delta_{3}=\epsilon^{2} F_{3 / 2}^{2}\left[\frac{1}{\left(F_{3 / 2}-F_{5 / 2}\right)}+\frac{1}{\left(F_{3 / 2}(1 \pm \epsilon)-F_{1 / 2}\right)}\right],
$$

where we may keep only the second- and third-order terms. This gives us the position of the third gap $\lambda_{-}^{(3)}<\lambda<\lambda_{+}^{(3)}$ :

$$
\begin{aligned}
\lambda_{ \pm}^{(3)}= & F_{3 / 2}+\epsilon^{2} F_{3 / 2}^{2}\left[\frac{1}{\left(F_{3 / 2}-F_{5 / 2}\right)}+\frac{1}{\left(F_{3 / 2}-F_{1 / 2}\right)}\right] \\
& \pm \frac{\epsilon^{3} F_{3 / 2}^{3}}{\left(F_{3 / 2}-F_{1 / 2}\right)^{2}} .
\end{aligned}
$$

Analogously, the gaps at integer $\nu$ 's can be considered. These are determined by the system

$$
\begin{aligned}
\left(\lambda-F_{0}\right) \Psi_{0}+\lambda \epsilon\left( \pm \Psi_{1}+\Psi_{1}\right) & =0 \\
\left(\lambda-F_{1}\right) \Psi_{1}+\lambda \epsilon\left(\Psi_{0}+\Psi_{2}\right) & =0 \\
\left(\lambda-F_{2}\right) \Psi_{2}+\lambda \epsilon\left(\Psi_{1}+\Psi_{3}\right) & =0
\end{aligned}
$$

For instance, position of the second gap in second order is given by the formulas

$$
\lambda_{-}^{(2)}=F_{1}-\frac{\epsilon^{2} F_{1}^{2}}{F_{2}-F_{1}}, \quad \lambda_{+}^{(2)}=F_{1}\left(1+2 \epsilon^{2}\right)-\frac{\epsilon^{2} F_{1}^{2}}{F_{2}-F_{1}} .
$$

\section{EXACT THEORY}

In exact nonlinear theory, the shape of free boundary is given in parametric form by a compound function $z(\zeta(u, t))$, where $z(\zeta)$ is a known function completely determined by the bottom shape [for example, $z(\zeta)=h\left(\sqrt{(\zeta+i)^{2}-(b / h)^{2}}-i\right)$ corresponds to a narrow vertical barrier of the height $b$ at $x=0$ on the straight horizontal bottom with the depth $y=-h$. The unknown function $\zeta(w, t)$ should be analytical in the stripe $-1<\operatorname{Im} w<0$ and the combination $[\zeta(u-i, t)+i]$ should take real values. These conditions relate the real and the imaginary parts of $\zeta(u, t)$ at the real axis [38]:

$$
\zeta(u, t)=u+(1+i \hat{R}) \rho(u, t),
$$

where $\rho(u, t)$ is a real function, and the linear anti-Hermitian operator $\hat{R}$ is $i \tanh k$ in Fourierrepresentation. In $u$-representation

$$
\hat{R} \rho(u, t)=\mathrm{P} . \mathrm{V} . \int_{-\infty}^{+\infty} \frac{\rho(\tilde{u}, t) d \tilde{u}}{2 \sinh [(\pi / 2)(\tilde{u}-u)]}
$$


The inverse operator $\hat{R}^{-1}=\hat{T}=-i \operatorname{coth} \hat{k}$ acts as

$$
\hat{T} \rho(u, t)=\mathrm{P} . \mathrm{V} \cdot \int_{-\infty}^{+\infty} \frac{\rho(\tilde{u}, t) d \tilde{u}}{1-\exp [\pi(\tilde{u}-u)]} .
$$

Note that the previously considered operator $[\hat{k} \tanh \hat{k}]$ is $-\hat{R} \partial_{u}$. The kinetic energy functional is now exactly equal to the expression at the r. h. s. of Eq. (6). The Lagrangian for $\psi(u, t)$ and $\zeta(u, t)$ is given by the formula

$$
\begin{aligned}
& \mathcal{L}_{\text {exact }}=\int\left|z^{\prime}(\zeta)\right|^{2}\left(\frac{\bar{\zeta}_{u} \zeta_{t}-\zeta_{u} \bar{\zeta}_{t}}{2 i}\right) \psi d u \\
& +\frac{1}{2} \int \psi \hat{R} \psi_{u} d u-\frac{g}{2} \int\{\operatorname{Im} z(\zeta)\}^{2} \operatorname{Re}\left(z^{\prime}(\zeta) \zeta_{u}\right) d u \\
& +\int \Lambda\left[\frac{\zeta-\bar{\zeta}}{2 i}-\hat{R}\left(\frac{\zeta+\bar{\zeta}}{2}-u\right)\right] d u
\end{aligned}
$$

where the (real) Lagrangian indefinite multiplier $\Lambda(u, t)$ has been introduced in order to take into account the analytical properties of the function $\zeta$ given by Eq.(38). From the above Lagrangian one can obtain the equations of motion. Variation of the action $\int \mathcal{L}_{\text {exact }} d t$ by $\delta \psi(u, t)$ gives the equation

$$
\left|z^{\prime}(\zeta)\right|^{2}\left(\bar{\zeta}_{u} \zeta_{t}-\zeta_{u} \bar{\zeta}_{t}\right) /(2 i)=-\hat{R} \psi_{u},
$$

which can be easily transformed to the form (compare with [38])

$$
\zeta_{t}=-\zeta_{u}(\hat{T}+i)\left[\frac{\hat{R} \psi_{u}}{\left|z^{\prime}(\zeta)\right|^{2}\left|\zeta_{u}\right|^{2}}\right]
$$

The variation of the action by $\delta \zeta(u, t)$ results after simplifying in the equation

$$
\left|z^{\prime}(\zeta)\right|^{2}\left\{\psi_{t} \bar{\zeta}_{u}-\psi_{u} \bar{\zeta}_{t}+g \bar{\zeta}_{u} \operatorname{Im} z(\zeta)\right\}-(1+i \hat{R}) \Lambda=0 .
$$

Since the product $\zeta_{u}(1+i \hat{R}) \Lambda$ has the same analytical properties as both $\zeta_{u}$ and $(1+i \hat{R}) \Lambda$, we can multiply Eq.(44) by $\zeta_{u}$ and write

$$
\left|z^{\prime}(\zeta)\right|^{2}\left\{\left[\psi_{t}+g \operatorname{Im} z(\zeta)\right]\left|\zeta_{u}\right|^{2}-\psi_{u} \bar{\zeta}_{t} \zeta_{u}\right\}-(1+i \hat{R}) \tilde{\Lambda}=0,
$$

where $\tilde{\Lambda}$ is another real function. The imaginary part of the above equation together with Eq.(42) result in

$$
\tilde{\Lambda}=-\hat{T}\left[\psi_{u} \hat{R} \psi_{u}\right] .
$$

Using this equality, we can reduce the real part of Eq. (45) to the form

$$
\psi_{t}+g \operatorname{Im} z(\zeta)=-\psi_{u} \hat{T}\left[\frac{\hat{R} \psi_{u}}{\left|z^{\prime}(\zeta)\right|^{2}\left|\zeta_{u}\right|^{2}}\right]-\frac{\hat{T}\left[\psi_{u} \hat{R} \psi_{u}\right]}{\left|z^{\prime}(\zeta)\right|^{2}\left|\zeta_{u}\right|^{2}},
$$

which is the Bernoulli equation in the conformal variables. Exact equations (43) and (47) [with given analytical function $z(\zeta)$ and with the condition (38)] completely determine the evolution of gravitational surface waves over the undulating bottom parameterized by a real parameter $r$ as $X_{b}(r)+i Y_{b}(r)=\left.z(\zeta)\right|_{\zeta=r-i}$.

Equations (43) and (47) can be represented in another form by using the identity $2 \hat{T}\left[\psi_{u} \hat{R} \psi_{u}\right]=\psi_{u}^{2}-\left(\hat{R} \psi_{u}\right)^{2}$ [38] and introducing the complex potential

$$
\Phi(u, t)=(1+i \hat{R}) \psi(u, t)
$$

(which is analytically continued to the stripe $-1<$ $\operatorname{Im} w<0)$ :

$$
\begin{aligned}
\zeta_{t}= & -\zeta_{u}(\hat{T}+i)\left[\frac{\operatorname{Im} \Phi_{u}}{\left|z^{\prime}(\zeta)\right|^{2}\left|\zeta_{u}\right|^{2}}\right], \\
\Phi_{t}= & -\Phi_{u}(\hat{T}+i)\left[\frac{\operatorname{Im} \Phi_{u}}{\left|z^{\prime}(\zeta)\right|^{2}\left|\zeta_{u}\right|^{2}}\right] \\
& -(1+i \hat{R})\left[\frac{\left|\Phi_{u}\right|^{2}}{2\left|z^{\prime}(\zeta)\right|^{2}\left|\zeta_{u}\right|^{2}}+g \operatorname{Im} z(\zeta)\right] .
\end{aligned}
$$

A very interesting point is that one can re-write equations (43) and (47) without the intermediate function $\zeta(u, t)$, but directly for $z(u, t)$. Indeed, after multiplying Eq. (43) by $z^{\prime}(\zeta)$ we obtain the equations

$$
\begin{gathered}
z_{t}=-z_{u}(\hat{T}+i)\left[\frac{\hat{R} \psi_{u}}{\left|z_{u}\right|^{2}}\right], \\
\psi_{t}+g \operatorname{Im} z=-\psi_{u} \hat{T}\left[\frac{\hat{R} \psi_{u}}{\left|z_{u}\right|^{2}}\right]-\frac{\hat{T}\left[\psi_{u} \hat{R} \psi_{u}\right]}{\left|z_{u}\right|^{2}},
\end{gathered}
$$

that is exactly the same system as was derived in [38] for a straight horizontal bottom. However, in our case analytical properties of the function $z(w, t)$ are different:

$$
\operatorname{Im} z(u) \neq \hat{R}[\operatorname{Re}(z(u)-u)] .
$$

The only requirements for $z(w, t)$ now are that it should be analytical in the stripe $-1<\operatorname{Im} w<0$ and the corresponding mapping should have a physical sense (no self-intersections are allowed). The question may arise: Where is the bottom shape in Eqs. (51)-(52)? The answer is simple: The shape of the bottom is an integral of motion for this system. Roughly speaking, each particular solution of Eqs. (51)-(52) corresponds to a flow over a definite topography determined by the initial condition $z(r-i, 0)$.

Analogously, Eqs. (49)-(50) can be represented as

$$
z_{t}=-z_{u}(\hat{T}+i)\left[\frac{\operatorname{Im} \Phi_{u}}{\left|z_{u}\right|^{2}}\right]
$$

$$
\Phi_{t}=-\Phi_{u}(\hat{T}+i)\left[\frac{\operatorname{Im} \Phi_{u}}{\left|z_{u}\right|^{2}}\right]-(1+i \hat{R})\left[\frac{\left|\Phi_{u}\right|^{2}}{2\left|z_{u}\right|^{2}}+g \operatorname{Im} z\right] .
$$




\section{NUMERICAL EXPERIMENTS}

\section{A. Different forms of equations}

For numerical simulations, still other equivalent forms of exact equations may be useful, since numerical stability depends dramatically on the choice of dynamical variables $39,40,41,42$. Two alternative sets of equations were used in computations presented below. First, as it was pointed in 39] for the case of deep water, a good practical choice for the dynamical variables is $A=1 / z_{u}$ and $B=\Phi_{u} / z_{u}$. It is easy to derive the equations of motion for $A(u, t)$ and $B(u, t)$ from Eqs. (54)-(55), and they are very elegant (compare with [39]):

$$
\begin{gathered}
A_{t}=-A_{u}(\hat{T}+i) \operatorname{Im}(B \bar{A})+A(\hat{T}+i) \partial_{u} \operatorname{Im}(B \bar{A}), \\
B_{t}=-B_{u}(\hat{T}+i) \operatorname{Im}(B \bar{A})-A(1+i \hat{R})\left[\partial_{u} \frac{|B|^{2}}{2}+g \operatorname{Im} \frac{1}{A}\right] .
\end{gathered}
$$

The variables $A$ and $B$ do allow stable numerical simulations for waves over varying seabed. However, analytical properties of $A$ and $B$ are not restricted by conditions similar to Eq. (38), and therefore the shape of the bottom is preserved in this case only approximately.

The second set of variables, that were used in numerical experiment, consists of two complex functions: $\zeta(u, t)$ and

$$
\beta(u, t)=\Phi_{u}(u, t) / \zeta_{u}(u, t),
$$

both having effectively controlled analytical properties. With this choice, the bottom shape is preserved exactly, but the corresponding equations of motion are slightly less compact:

$$
\begin{aligned}
\zeta_{t}= & -\zeta_{u}(\hat{T}+i) \operatorname{Im}\left(\frac{\beta}{\left|z^{\prime}(\zeta)\right|^{2} \bar{\zeta}_{u}}\right) \\
\beta_{t}= & -\beta_{u}(\hat{T}+i) \operatorname{Im}\left(\frac{\beta}{\left|z^{\prime}(\zeta)\right|^{2} \bar{\zeta}_{u}}\right) \\
& -\zeta_{u}^{-1}(1+i \hat{R}) \partial_{u}\left[\frac{|\beta|^{2}}{2\left|z^{\prime}(\zeta)\right|^{2}}+g \operatorname{Im} z(\zeta)\right]
\end{aligned}
$$

It is necessary to explain here some important details about space-periodic solutions of the system (59)-(60), since spectral numerical methods deal with periodic functions. Such solutions exist if the function $z^{\prime}(\zeta)$ is periodic with a fixed real period $L$, so that $z(\zeta+L)=L+z(\zeta)$. However, this does not imply that the functions $\zeta_{u}(u, t)$ and $\beta(u, t)$ have a fixed $u$-period. It would be so, but the linear operator $\hat{T}$ is singular at small $k$, and its action on a constant function is not periodic in $u$ : $\hat{T} C=C u$. Thus in the right-hand-sides of the Eqs. (59)- (60) we have non-periodic terms. Therefore $\zeta_{t}(u, t)$ and $\beta_{t}(u, t)$ cannot retain a constant $u$-period. However, $\zeta_{t}(u, t)$ and $\beta_{t}(u, t)$ can be space-periodic with a time-dependent $u$-period. So, at arbitrary moment of time we will have the equality $\zeta(u, t)+i=(L / 2 \pi) \zeta_{*}(2 \pi \alpha(t) u / L, t)$, where

$$
\begin{aligned}
\zeta_{*}(\vartheta, t) & =\vartheta+i \alpha(t)+\sum_{m=-\infty}^{+\infty} \frac{2 \rho_{m}(t) \exp (i m \vartheta)}{1+\exp (2 m \alpha(t))} \\
& =\vartheta+i \alpha(t)+\left(1+i \hat{\mathrm{R}}_{\alpha}\right) \rho(\vartheta, t),
\end{aligned}
$$

with an unknown real function $\alpha(t)$. The unknown complex Fourier coefficients $\rho_{m}(t)$ correspond to a real $(2 \pi$ periodic on the variable $\vartheta$ ) function $\rho(\vartheta, t)$ :

$$
\rho(\vartheta, t)=\sum_{m=-\infty}^{+\infty} \rho_{m}(t) \exp (i m \vartheta), \quad \rho_{-m}(t)=\bar{\rho}_{m}(t) .
$$

The linear operator $\hat{\mathrm{R}}_{\alpha}$ is diagonal in the discrete Fourier representation: $\mathrm{R}_{\alpha}(m)=i \tanh (\alpha m)$.

Analogously, $\beta(u, t)$ can be represented as $\beta=$ $(g L /(2 \pi))^{1 / 2} \beta_{*}(\vartheta, t)$, where

$$
\beta_{*}(\vartheta, t)=\sum_{m=-\infty}^{+\infty} \frac{2 \chi_{m}(t) \exp (i m \vartheta)}{1+\exp (2 m \alpha(t))}=\left(1+i \hat{\mathrm{R}}_{\alpha}\right) \chi(\vartheta, t)
$$

Equations of motion for the real functions $\alpha(t), \rho(\vartheta, t)$, and $\chi(\vartheta, t)$ follow from Eqs. (59)- (60):

$$
\begin{aligned}
\dot{\alpha}(t) & =\frac{1}{2 \pi} \int_{0}^{2 \pi} \operatorname{Im}\left(\frac{-\beta_{*}(\vartheta)}{\left|z_{*}^{\prime}\left(\zeta_{*}\right)\right|^{2} \bar{\zeta}_{*}^{\prime}(\vartheta)}\right) d \vartheta \\
\dot{\rho}(\vartheta, t) & =\operatorname{Re}\left(\zeta_{*}^{\prime}\left(\hat{\mathrm{T}}_{\alpha}+i\right) \operatorname{Im}\left(\frac{-\beta_{*}(\vartheta)}{\left|z_{*}^{\prime}\left(\zeta_{*}\right)\right|^{2} \bar{\zeta}_{*}^{\prime}(\vartheta)}\right)\right), \quad(64) \\
\dot{\chi}(\vartheta, t) & =\operatorname{Re}\left(\beta_{*}^{\prime}\left(\hat{\mathrm{T}}_{\alpha}+i\right) \operatorname{Im}\left(\frac{-\beta_{*}(\vartheta)}{\left|z_{*}^{\prime}\left(\zeta_{*}\right)\right|^{2} \bar{\zeta}_{*}^{\prime}(\vartheta)}\right)\right. \\
& \left.-\frac{1}{\zeta_{*}^{\prime}}\left(1+i \hat{\mathrm{R}}_{\alpha}\right) \partial_{\vartheta}\left[\frac{\left|\beta_{*}\right|^{2}}{2\left|z_{*}^{\prime}\left(\zeta_{*}\right)\right|^{2}}+\operatorname{Im} z_{*}\left(\zeta_{*}\right)\right]\right)(65)
\end{aligned}
$$

where $z_{*}\left(\zeta_{*}\right)=(2 \pi / L) z\left(-i+L \zeta_{*} /(2 \pi)\right), \zeta_{*}^{\prime}=\partial_{\vartheta} \zeta_{*}$ and so on. The linear operator $\hat{\mathrm{T}}_{\alpha}$ is regular. In the discrete Fourier representation it is defined as follows:

$$
\begin{aligned}
\mathrm{T}_{\alpha}(m) & =-i \operatorname{coth}(\alpha m), & & m \neq 0 \\
& =0, & & m=0 .
\end{aligned}
$$

Two numerical experiments are briefly reported below, first of them employing Eqs.(56)-(57), and the second one employing Eqs.(59)-(60). Both the systems (56)-(57) and (59)-(60) are equally convenient for numerical solution by spectral methods, inasmuch as the multiplications can be performed in $u$-representation while the linear operators $\hat{R}$ and $\hat{T}$ (also the $u$-differentiation) are simple in the Fourier representation. Efficient subroutine libraries for the fast Fourier transform are now available. The integration schemes in both cases were based on the RungeKutta 4-th order method, similarly to work [40]. For computing the discrete Fourier transform, the FFTW library was used [3]. The length scale was normalized by a factor $h=L /(2 \pi)$ and the velocity scale by $(g h)^{1 / 2}$. 


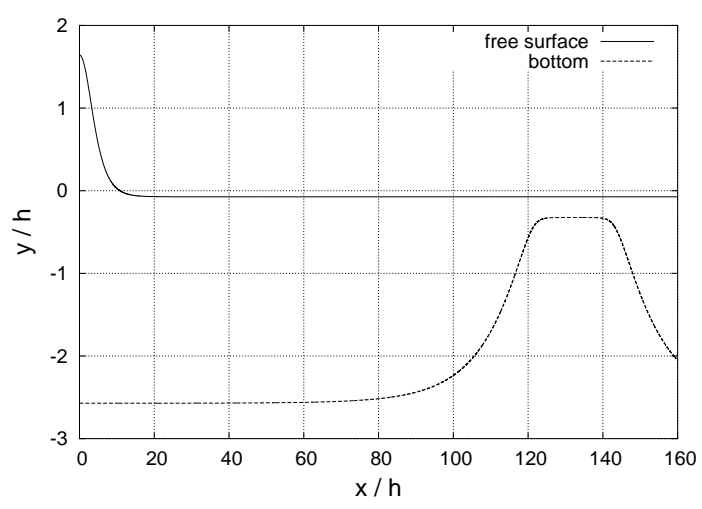

FIG. 3: (i) Free surface and bottom for $t=0$. Only part of the entire periodic domain is shown.
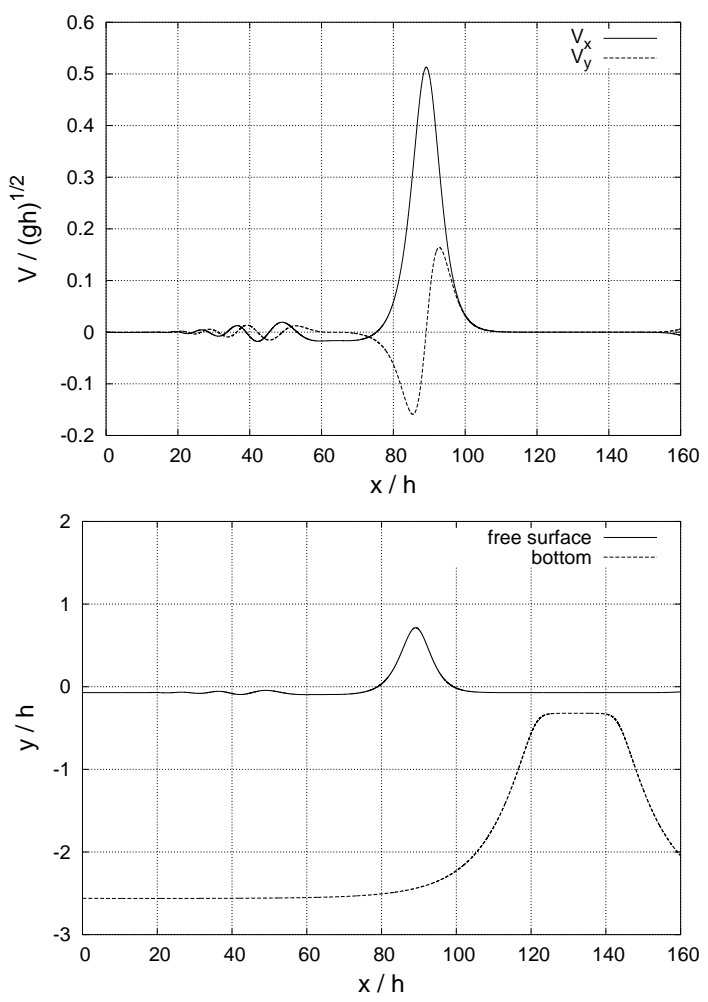

FIG. 4: (i) $t=50$ : propagation stage.

\section{B. Numerical results for Eqs.(56)-(57)}

The above remark about time-dependent $u$-period equally concerns the system (56)-(57) as (59)-(60). However, for localized disturbances the relative change of $\alpha(t)$ remains small. Therefore solitary waves are possible to simulate with a constant $\alpha$ and with the regularized $\hat{T}$. It was done so the first numerical experiment [referred as (i)], where periodic boundary conditions were applied with the fixed $u$-period $L=200$. The initial conditions
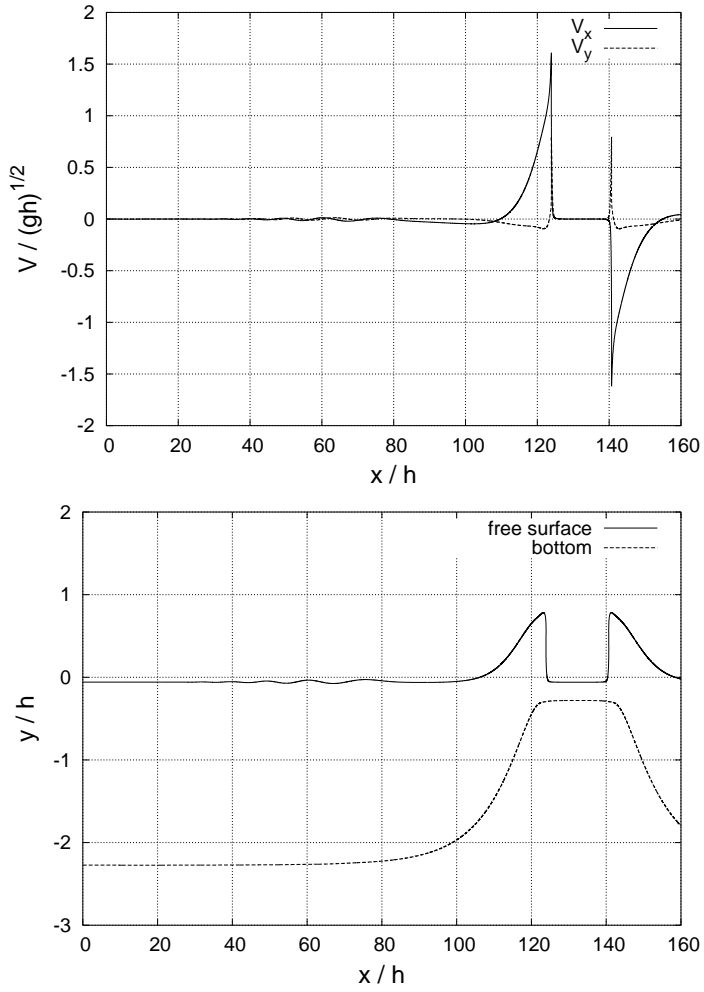

FIG. 5: (i) $t=69$ : breaking of the wave.

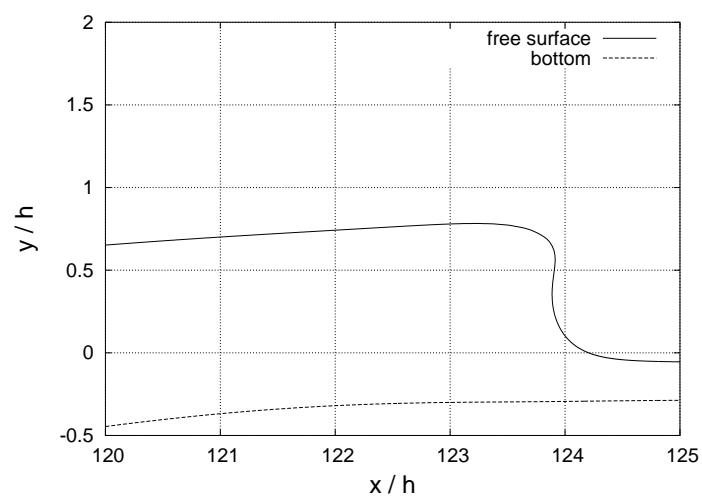

FIG. 6: (i) Crest of the wave for $t=69$.

were taken $B(u, 0)=0$ and

$$
\frac{1}{A(u, 0)}=z_{u}(u, 0)=\frac{2.5 E(u)+0.25}{E(u)+1}+\frac{1.4}{C(u)},
$$

where

$$
\begin{aligned}
& E(u)=\exp [8.0(\cos (2 \pi u / L)-0.1)] \\
& C(u)=\cosh [(L / \pi) \sin \{\pi(u+i) / L\}] .
\end{aligned}
$$

This initial configuration is symmetric (even). It results after some time in two oppositely propagating, nearly solitary waves. The waves are created in the region where 


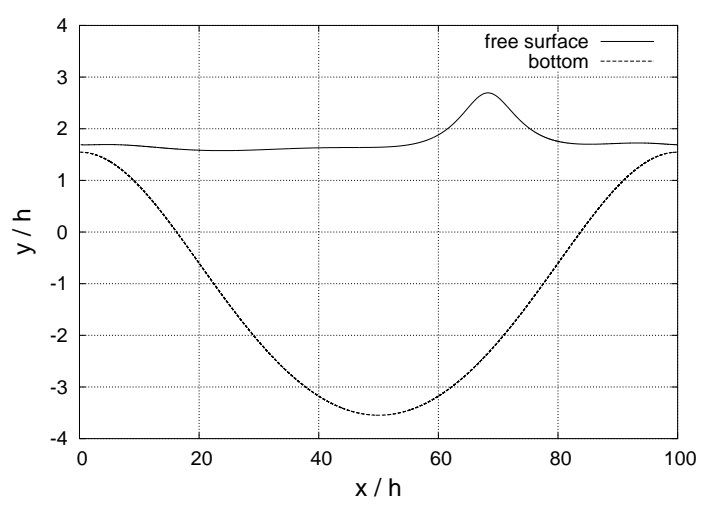

FIG. 7: (ii) The bottom profile and shape of the free surface at $t=0$. The velocity field is everywhere zero.

the depth is maximal. In the course of motion each wave approaches a shallow region where the surface profile $y=$ $\eta(x, t)$ becomes steeper and finally multi-valued.

Some of the results of this numerical experiment are presented in Figs 3.6] where the velocity distribution on the surface is shown, as well as shapes of free surface and of the bottom for several moments of time. In general, the computed wave profiles look quite realistic, though the present theory does not take into account viscous effects. Steeping of the wave profile is clearly seen. It should be noted, however, in these simulations the bottom shape is preserved only approximately due to discretization errors, and the same concerns the total energy. The computation was stopped well before the moment of formation of a singularity on the crests of the waves, when the numerical scheme becomes invalid. In real world this moment corresponds to development of a three-dimensional instability resulting in vortices, splash and foam.

\section{Numerical results for Eqs.(59)- 60}

In the second numerical experiment [referred as (ii)], the shape of the bottom was fixed by analytical function

$$
z(\zeta)=\zeta+i \frac{L d}{2 \pi} \exp (2 \pi i(\zeta+i) / L)
$$

with the dimensionless parameters $L=100, d=0.16$. The initial velocity field was taken zero: $\beta(u, 0)=0$, while $\zeta(u, 0)$ had the form

$\zeta(u, 0)=u+\Theta(0.63,0.0, u+i)+0.06 \Theta(0.9,0.04, u+i)$,

where

$$
\Theta(r, p, w) \equiv-i \frac{L}{2 \pi} \ln \left(\frac{1+r \exp (-2 \pi i(w-p L) / L)}{1+r \exp (+2 \pi i(w-p L) / L)}\right) .
$$

Qualitatively, these initial conditions are similar to those in the first experiment, however now two oppositely propagating waves are created over inclined region of the bottom, so there is no left-right symmetry in the evolution.
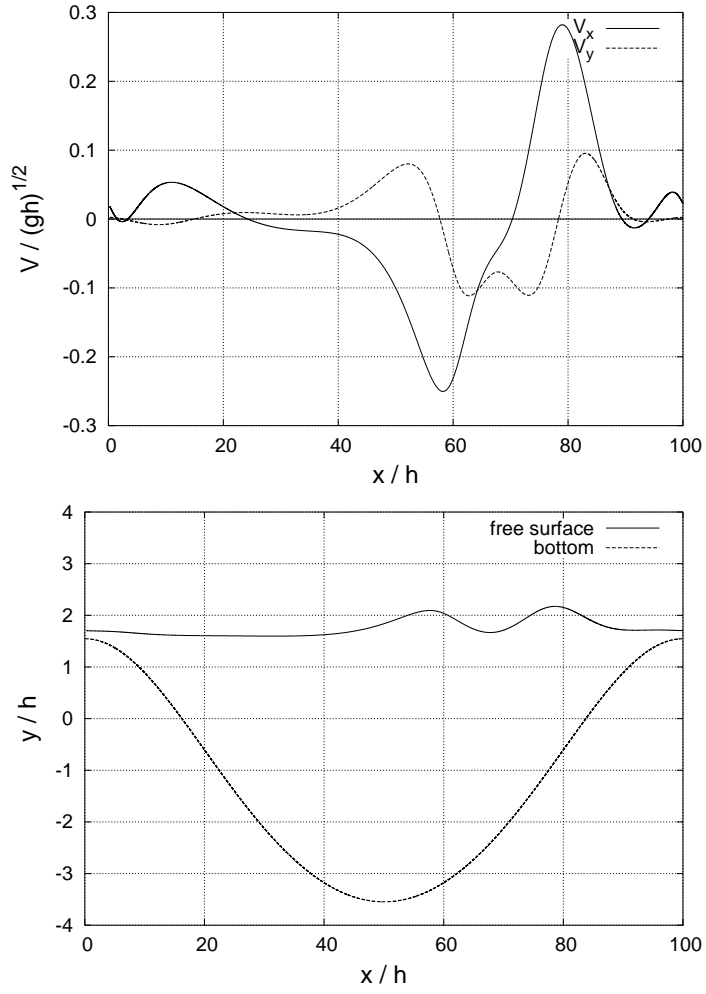

FIG. 8: (ii) Velocity distribution on free surface and shape of the surface at $t=6$.
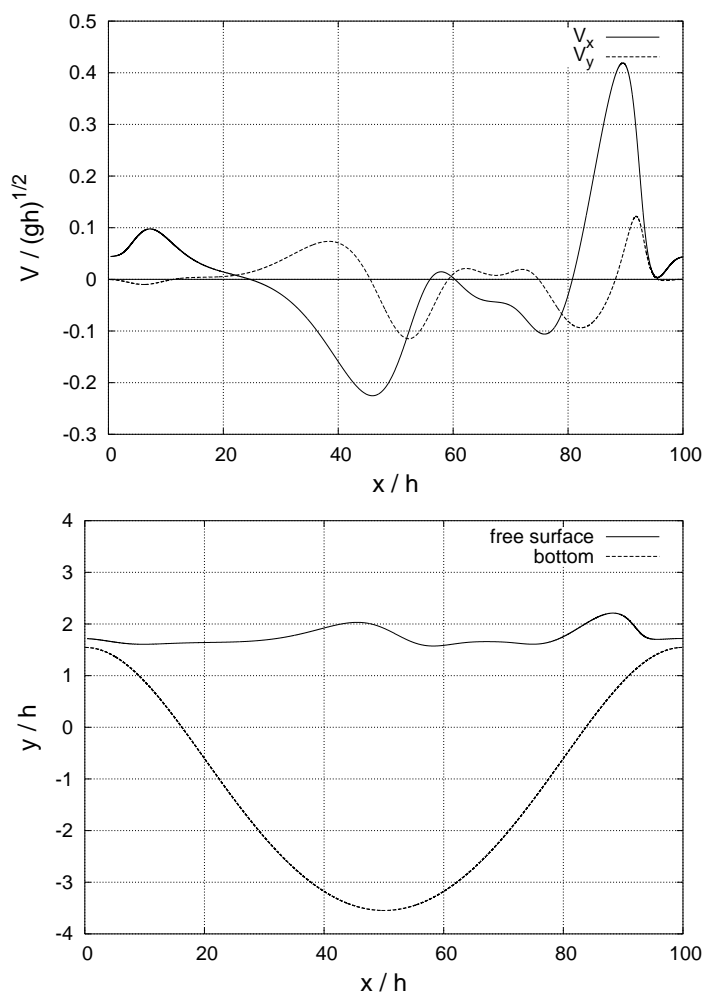

FIG. 9: (ii) The same as in Fig 8 at $t=12$. 

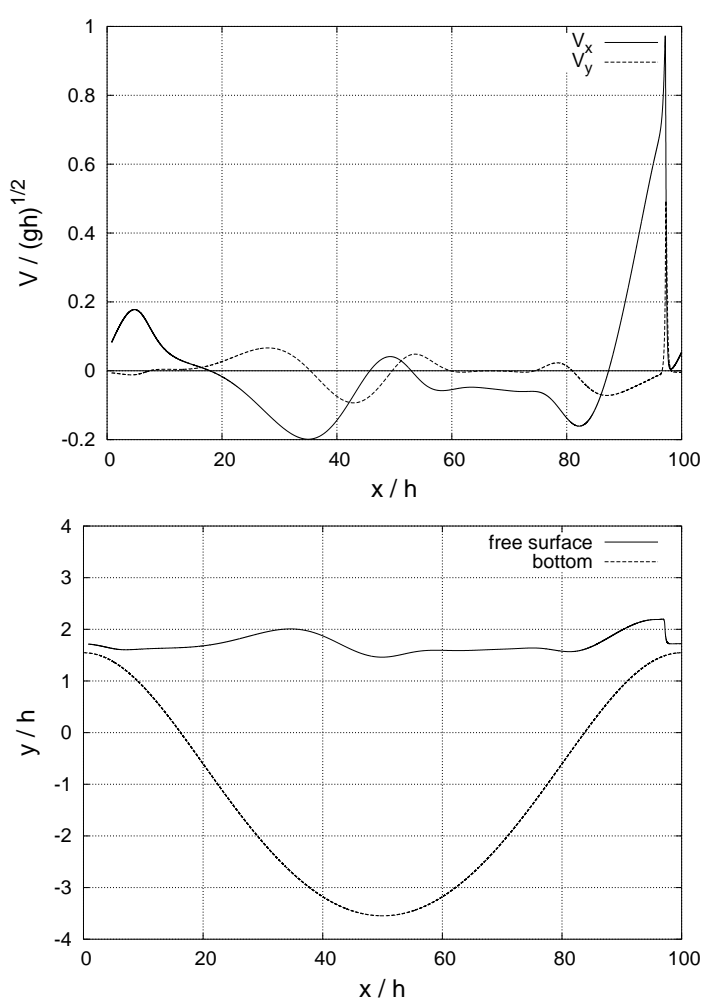

FIG. 10: (ii) The same as in Fig[ at $t=17$.

The full system (63)- (65) was solved with a high accuracy for $m$ in the limits $-7000<m<7000$ (the energy conservation was up to 12 digits for a long "smooth" initial stage of the evolution, and it was up to 5 digits for the final stage just before the breaking). This more accurate numerical solution was compared to a less accurate solution obtained with fixed $\alpha=\alpha(0)$. The difference was found very small.

The corresponding numerical results are presented in
Figs [7. 10 Here we again observe steeping of the wave profile with the tendency towards finite time singularity formation on the crest. Such behavior indeed takes place in natural conditions when the flows are almost two-dimensional.

\section{SUMMARY}

In this paper we have derived approximate weaklynonlinear, as well as exact nonlinear equations of motion for potential water waves over a strongly inhomogeneous bottom. The consideration was based on using the conformal mappings. For linear waves over periodic seabed, the band structure of the spectrum has been calculated.

Though the obtained exact equations can be written in formally the same form as those derived in [38] for a straight horizontal bottom, but admissible solutions have different analytical properties if the bottom is inhomogeneous. When the equations are written in this form, the bottom shape is preserved as an integral of motion.

Numerical experiments have been carried out that confirm advantage of the theory by giving quite realistic pictures for wave profiles before wave breaking.

Of course, the above "inviscid theory" works only on large enough spatial scales and only until the singularity moment, as it was clear from the very beginning. Practically, this theory is good for description sea and ocean waves before their breaking.

\section{Acknowledgments}

These investigations were supported by the INTAS under grant number 00292, by RFBR, by the Russian State Program for Support of the Leading Scientific Schools, and by the Russian Science Support Foundation.
[1] H. Lamb, Hydrodynamics (Cambridge University Press, Cambridge, 1995).

[2] R. S. Johnson, Proc. Camb. Phil. Soc. 73, 183 (1973).

[3] S. Leibovich and J. D. Randall, J. Fluid Mech. 53, 481 (1973).

[4] R. S. Johnson, J. Fluid Mech. 60, 813 (1973).

[5] L. Q. Spielvogel, J. Fluid Mech. 74, 685 (1975).

[6] C. Lozano and R. E. Meyer, The Physics of Fluids 19, 1075 (1976).

[7] I. A. Svendsen and J. B. Hansen, J. Fluid Mech. 87, 433 (1978).

[8] J. W. Miles, J. Fluid Mech. 91, 181 (1979).

[9] A. D. Heathershaw, Nature 296, 343 (1982).

[10] C.C. Mei, J. Fluid Mech. 152, 315 (1985).

[11] J. Miles and R. Salmon, J. Fluid Mech. 157, 519 (1985).

[12] J.T. Kirby, J. Fluid Mech. 162, 171 (1986); http://chinacat.coastal.udel.edu/ kirby/
[13] T. Hara and C.C. Mei, J. Fluid Mech. 178, 221 (1987).

[14] C.C. Mei, T. Hara, and M. Naciri, J. Fluid Mech. 186, 147 (1988).

[15] A. G. Davies, E. Guazzelli, and M. Belzons, Phys. Fluids A 1, 1331 (1989).

[16] A. Falques, V. Iranzo, and A. Montoto, Phys. Fluids A 5, 3071 (1993).

[17] P. L.-F. Liu and H. H. Yeh, Phys. Fluids 8, 2060 (1996).

[18] U. Putrevu and J. Oltman-Shay, Phys. Fluids 10, 330 (1998).

[19] P. L.-F. Liu, H. Yeh, P. Lin, K.-T. Chang, and Y.-S. Cho, Phys. Fluids 10, 1635 (1998).

[20] K. M. Mok and H. Yeh, Phys. Fluids 11, 2906 (1999).

[21] J. W. Miles and P. G. Chamberlain, J. Fluid Mech. 361, 175 (1998).

[22] J. Miles, J. Fluid Mech. 376, 53 (1998).

[23] Y. Liu and D. K. P. Yue, J. Fluid Mech. 356, 297 (1998). 
[24] P. Milewski, Physica D 123, 36 (1998).

[25] G. A. Athanassoulis and K. A. Belibassakis, J. Fluid Mech. 389, 275 (1999).

[26] Y. Agnon, Phys. Rev. E 59, R1319 (1999).

[27] M. Torres, J. P. Adrados, F. R. Montero de Espinosa, D. Garcia-Pablos, and J. Fayos, Phys. Rev. E 63, 011204 (2000).

[28] J. H. Pihl, C. C. Mei, and M. J. Hancock, Phys. Rev. E 66016611 (2002).

[29] Zhen Ye, Phys. Rev. E 67036623 (2003).

[30] R. Porter and D. Porter, J. Fluid Mech. 483, 129 (2003).

[31] L. D. Landau and E. M. Lifshitz, Quantum Mechanics (Pergamon, Oxford, 1977).

[32] V. E. Zakharov, Prikl. Mekh. Tekh. Fiz. 2, 86 (1968).

[33] V. E. Zakharov and E. A. Kuznetsov, Usp. Fiz. Nauk 167, 1037 (1997) [Phys. Usp. 40, 1087 (1997)].

[34] L. V. Ovsyannikov, Dynamika Sploshnoi Sredy, Lavrentiev Institute of Hydrodynamics Sib. Branch of Ac. Sci. USSR, No. 15, pp. 104-125 (1973) [in Russian].
[35] A. I. Dyachenko, E. A. Kuznetsov, M. D. Spector, and V. E. Zakharov, Phys. Lett. A 221, 73 (1996).

[36] A. I. Dyachenko, Y. V. L'vov, and V. E. Zakharov, Physica D 87, 233 (1995).

[37] V. E. Zakharov and A. I. Dyachenko, Physica D 98, 652 (1996).

[38] A. I. Dyachenko, V. E. Zakharov, and E. A. Kuznetsov, Fiz. Plazmy 22, 916 (1996) [Plasma Phys. Rep. 22, 829 (1996)].

[39] A. I. Dyachenko, Doklady Akademii Nauk 376, 27 (2001) [Doklady Mathematics 63, 115 (2001)].

[40] V. E. Zakharov, A. I. Dyachenko, and O. A. Vasilyev, European Journal of Mechanics B/Fluids 21, 283 (2002).

[41] A. I. Dyachenko, to be published (2004).

[42] V. E. Zakharov and P. M. Lushnikov, to be published (2004).

[43] http://www.fftw.org/ 